\title{
Set of Methods for Spontaneous ICH Segmentation and Tracking from CT Head Images
}

\author{
Noel Pérez ${ }^{1}$, José A. Valdés ${ }^{1}$, Miguel A. Guevara ${ }^{1}$, Luis A. Rodríguez², \\ and J. M. Molina ${ }^{1}$ \\ ${ }^{1}$ Center for Advanced Computer Sciences Technologies, \\ Ciego de Ávila University,Ciego de Ávila, Cuba \\ \{noel, jose, miguel, juanmiguelmolina\} \\ ainformatica.unica.cu \\ ${ }^{2}$ Intensive Care Unit, Morón Hospital, Ciego de Ávila, Cuba \\ lars@trocha.cav.sld.cav.cu
}

\begin{abstract}
Spontaneous intracerebral hemorrhage (ICH) is a common cause of stroke, due to this; the early evolution and quantitative analysis of the $\mathrm{ICH}$ is important for the treatment and the course of patient's recovery. Computerbased diagnosis systems have played an important role in quantitative analysis of medical images aiding medical personnel in selecting the appropriated treatment of diseases. This paper outlines a set of three methods for ICH segmentation and tracking from computer tomography (CT) head images, based on a suitable combination of digital image processing and pattern recognition techniques. Two of these methods are carried out in a semiautomatic way and the other one is performed in a manual way. Methods developed were tested successfully by medical researchers in a representative dataset of CT head images (patient studies).
\end{abstract}

Keywords: Intracerebral hemorrhage, medical images analysis, 3D mathematic morphology, segmentation and tracking, deformable models.

\section{Introduction}

Spontaneous Intracerebral hemorrhage (ICH) is a common cause of stroke, accounting for between 5 and $10 \%$ of all strokes. In a consecutive series of 938 stroke patients enrolled into the NINCDS Stroke Data Bank, primary ICH accounted for $10.7 \%$ of the cases. The age-adjusted annual incidence rates for primary intracerebral hemorrhage range from 11 to 31 per 100,000 populations in predominantly Caucasian population based-studies with a high rate of computer tomography (CT) scanning [1]. The early evolution and quantitative analysis of the human cerebrovascular illness are significant for the treatment and the course of patient's recovery. Computer-based diagnosis systems have played an important role in quantitative analysis of medical images aiding medical personnel in selecting the appropriated treatment of diseases [2].

Many approaches for ICH segmentation and tracking have been reported in the literature. Cosic and Loncaric [2, 3] proposed a method based on unsupervised fuzzy clustering and expert system-based procedure region labeling techniques that includes 
volume measurement of the ICH and the edema regions but not always the automatic method perform a correct segmentation. Majcenic and Loncaric [4] proposed a stochastic method for ICH segmentation based on simulated annealing techniques. However these methods are computationally complex.

This paper outlines a set of three methods for ICH segmentation and tracking from CT head images, two of these methods are performed in a semiautomatic way and the other one in manual way. One of the semiautomatic methods is focused mainly in 3D mathematic morphology operations and the other combine live wire and graphical information retrieval techniques. The manual method use mainly deformable models, but need a major user intervention although it demonstrated a more precise and accurate ICH segmentation. This method was included with the intention to evaluate the semiautomatic methods performance.

The set of methods was used by medical researchers to evaluate the behavior and changes of spontaneous ICH (shape, size, etc.) during the disease course and validated successfully in a representative dataset of 36 ICH CT head images patient studies.

The paper is organized as follows: section 2 outlines the implementation of the proposed set of methods. In section 3 we describe results obtained with the application of methods to a representative dataset of ICH CT head images patient studies. Conclusions and some ideas about the future work are showed in section 4 .

\section{Proposed Methods}

Developed methods for segmentation and tracking of spontaneous ICH from CT head images patient studies are based on suitable combinations of digital image processing and pattern recognition techniques. Included methods were:

1. 3D mathematical morphology based method.

2. Similarity based method.

3. Manual based method.

\subsection{D Mathematical Morphology Based Method}

This method relies on the application of 3D mathematical morphology, histogram analysis, optimal thresholding, and prior geometric information techniques. Each patient image study is considered as an anisotropic volume restricted manually to the most outer neighbor ICH slices, with the intention to increase the automation level of the segmentation process and to reduce the computational cost.

The computed tomography images have common intensities frequencies histogram, in that way, we can perform some important correlations between image objects of interest and the characteristics intensities level values of skull, grey matter and white matter. In our work was observed heuristically the ICH intensities frequencies histogram values are related to higher intensities, which was consider as the start point for image segmentation.

\subsubsection{Preprocessing}

The preprocessing remove short duration impulses and smooth lightly the image edges, applying a median filter slice by slice, in axial form, with a windows of $3 \times 3$ pixels. 


\subsubsection{ICH Auto-detection}

Autodetection consists in compute the global maximum, which is used as the threshold value for the ICH segmentation (to produce a binary mask). Hereafter 3D mathematical morphology operations are applied to autodetect the ICH.

Algorithm 1. Semiautomatic method based on 3D mathematical morphology.

1: Define ICH neighbor slices interval.

2: Create an anisotropic volume of interest (VOI) containing the ICH slices.

$\mathrm{St}=\left\{(x, y, z) \in N^{3}: 0<x<x_{f}, 0<y<y_{f}, z_{0}<z<z_{f}\right\} ; \quad \mathrm{i}=\left\{\mathrm{i}_{1}, \mathrm{i}_{2}, \mathrm{i}_{3} \ldots \mathrm{i}_{\mathrm{n}}\right\} \quad \mathrm{n}=255$

$x_{f}$ and $y_{f}$ means row and column image size, $z_{0}$ and $z_{f}$ are the most outer neighbor slices. The studies intensities are defined as one-dimensional array $i$ with $n$ intensity levels.

3: Compute $g m=\max (S t) ; \quad g m$ is the maximum intensity value of St.

4: Obtain ICH binary mask using gm as threshold value.

$$
\operatorname{ICHmask}_{(x, y, z)}=\left\{\begin{array}{cc}
0 & \forall \mathrm{St}_{(x, y, z)}<\mathrm{gm}^{*} 0.90 \\
1 & \forall \mathrm{St}_{(x, y, z)} \geq \mathrm{gm} * 0.90
\end{array}\right\}
$$

5: mer $=I C H m a s k \ominus s e:$ where $\ominus$ represent the morphological erosion operation and $s e$ is an spherical structuring element ( $s e$ diameter is set to 5 voxels and it was determine heuristically).

6: Select the fa_mask (biggest foreground connected object in $m e r$ ), which is a first approach of the ICH segmentation.

7: $I C H \_$mask $=f a \_m a s k \oplus s e: I C H \_$mask is the 3D ICH binary mask.

6: Apply a flood fill operation to avoid holes inside the $\mathrm{ICH}$ _mask.

7: Multiply the new ICH_mask by the original VOI and ICH segmentation is carried out.

\subsection{Similarity Based Method}

Similarity based method involved two main steps: pick a query object (ICH) and afterward made the automatic ICH retrieval on the remainder patient study.

\subsubsection{Select Query Object}

Query object selection consists in the extraction of the ICH contour. We used live wire techniques [5] to extract the object edges, and hereafter based on the selected contour are computed automatically the initials reference points with their associated similarity vectors. Reference points and their associated similarity vectors are the query basic elements. The selection of query object $\left(I_{n}\right)$ is made by the following steps:

1. Extract the ICH contour $\left(C T_{n}\right)$.

2. Extract the reference points (set of points that belong to query object contour)

3. Create similarity vectors, from reference points. 
Query object elements are formed by the reference points and their associated similarity vectors. The quantity of reference points and their associated similarity vectors is variable and depend of the specific problem domain knowledge.

2.2.1.1 ICH Contour Extraction. We used live wire techniques for object contour extraction (segmentation). Live wire is a recently proposed interactive boundary tracking technique [6] that share some similarities with snakes and it is generally considered in the literature as a competing snake technique. Like snakes, the idea behind the live wire technique is to allow image segmentation to occur with minimal user interaction, while at the same time allowing the user to exercise control over the segmentation process. This technique share two essential components: a local cost function that assigns lower cost to image features of interest, such as edges, and an expansion process that forms optimal boundaries for objects of interest based on the cost function and seed points provided interactively by the user [5]. The contour obtained after live wire application is a not continuous, product of this, we interpolate it with a spline function to obtain a continuous curve to produce a better object contour approximation. The extracted curve is use after to compute the reference points.

2.2.1.2 Reference Points. Reference points are formed by a set of points that are located close or in the object contour with direction $i \theta$, where $i: 0 . . \alpha-1$, $\alpha=\operatorname{int}(360 / \theta), 0<\theta \leq 180^{\circ}$ and $\theta$ is the displacement angle, taking the object mass center as the center of the coordinates system. The angle $\theta$ will be selected by the user and it depend of the problem knowledge domain (Fig. 1). Initial reference points are determined by the following algorithm:

Algorithm 2. Generate Initial Reference Points

1: Select initial slice $I(n)$

2: Select $\theta, 0<\theta \leq 180^{\circ}$

3: Select $\mathcal{E}$ : admissible (small) distance between $P_{i}(x, y)$ and $C T n$

4: Compute $P_{i}(x, y)$ : reference points

coordinates $(X, Y)$ of $P_{i}$ are obtained by the expression:

$P_{i}(X, Y)=C_{x}+r * \cos (i), C_{y}+r * \sin (i)$

where $r \in \mathrm{Z}^{+}, 1 \leq r \leq \mathrm{R}$ and $\mathrm{r}$ increasein 1 on each iteration until $\mathrm{r}=\mathrm{R}$ and $\mathrm{R}$ is the value in which $d_{P i}\left(P_{i}(X, Y), C T_{n}\right) \leq \mathcal{E}, \quad d$ is the Euclidian distance between $P_{i}(x, y)$ and $C T_{n}$

$C x$ and $C y$ are the $(X, Y)$ centroid coordinates

5: Store new founded reference points in the $P_{i}(x, y)$ array 


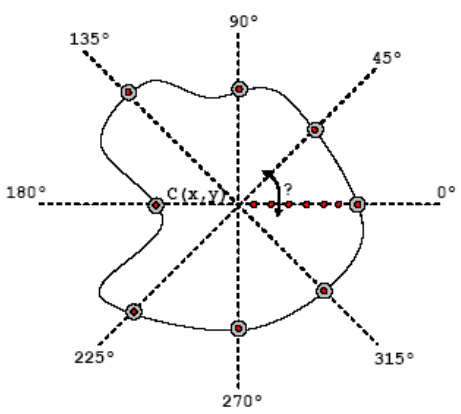

Fig. 1. Reference points obtained with $\theta=45^{\circ}, \varepsilon=0.5$

2.2.1.3 Similarity Vectors. Similarity vectors $\left(S_{i}\right)$ are the basic elements that allow to track the object on the rest of slices (images) belonging to a selected patient study. The reference points represent the centroid $\left(\mathrm{C}_{\mathrm{x}, \mathrm{y}}\right)$ of the similarity matrixes $\left(Q_{i}\right)$. Similarity matrixes are $M x M$ windows, where $M=2 * \delta+1, \delta$ is a positive integer scalar provided by the user (Fig. 2). The similarity vectors are obtained from the similarity matrixes using the following mathematic formulation:

$$
\begin{aligned}
& S_{i}=\left\{a_{1,1} \ldots a_{m, 1}, a_{1,2} \ldots a_{m, 2}, a_{1, m} \ldots a_{m, m}, a_{1, m+1} \ldots a_{m, m+1}\right\} \\
& \begin{array}{lllll}
a_{11} & a_{12} & a_{13} & \cdots & a_{1 m}
\end{array} \\
& \begin{array}{lllll}
a_{21} & a_{22} & a_{23} & \cdots & a_{2 m}
\end{array} \\
& Q_{i}=a_{31} \quad a_{32} \quad a_{33} \quad \cdots \quad a_{3 m} \\
& \begin{array}{lllll}
a_{m 1} & a_{m 2} & a_{m 3} & \cdots & a_{m m}
\end{array}
\end{aligned}
$$

where $S_{i}$ represent the set of similarity vectors corresponding to $P_{i}$ and $Q_{i}$.

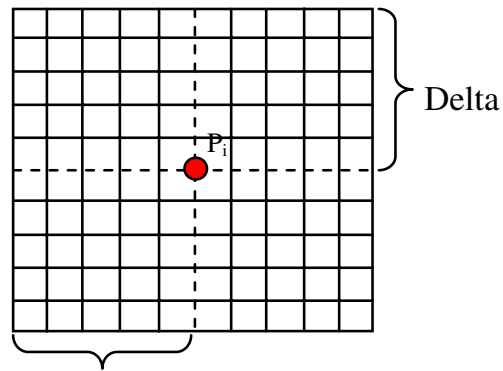

Delta

Fig. 2. Similarity matrix

\subsubsection{Object Retrieval}

Object retrieval (which is carried out in an automatic way) has the aim to track the query object picked over (user selected) slices (images) of patient study. This 
algorithm tries to find the points more similar to the current reference points on the slice under processing. The new founded points are used then as the reference points to detect the object in the next slice. This process is repeated over all the slices (selected by the user) that belong to the current patient study.

Object retrieval is in general a process devoted to find a query object on a set of images or images databases [7].

In our case object retrieval has the aim to track (extract) the $\mathrm{ICH}$ on a set of slices selected by the user that belong to the patient under study.

Similarity descriptors are critical for the performance of object retrieval process. Several descriptors were evaluated, but the best results were achieved with the distance equation (eq. 1) proposed by Fuertes [8], which we use to select the new reference points belonging to the slice under analysis.

$$
d\left(P_{i(n)}, P_{i(n+1)}\right)=\sqrt{\left(S_{i(n)}-S_{i(n+1)}\right)^{t} *\left(S_{i(n)}-S_{i(n+1)}\right)}
$$

$S_{i(n)}$ is a similarity vector associated to reference point $P_{i(n)}$ in the $I_{n}$ image, $S_{i(n+1)}$ is a vector associated to the point $P_{i(n+1)}$ in the $I_{n+1}$ slice, $t$ denote a transpose matrix. The new reference points will be those $P_{i(n+1)}$ where $d\left(P_{i(n)}, P_{i(n+1)}\right)$ is minimum.

The method employ the created similarity vectors on query object to find the reference points related to $\mathrm{ICH}$ : the points that identify the $\mathrm{ICH}$ occurrence on the rest of slices set of patient under study.

The object retrieval algorithm developed is outline below:

\section{Algorithm 3. Retrieval}

1: Select new slice $\left(I_{(n+1)}\right)$

2: Compute $P_{i k}$ points

for each $i$ angle

$$
P_{i k}(X, Y)=C_{n_{x}}+k * \cos (i), C_{n y}+k * \sin (i)
$$

where $k: 1 . . D+\sigma, \sigma$ is a value selected by the user, $D$ is the euclidian distance between $C_{n}$ and $P_{i(n)}$

Build vector $S_{i k}$ from $P_{i k}$ (see 2.2.1.3)

Compute $R_{i k}$

$$
R_{i k}=d\left(S_{i k}, S_{i n}\right): d \text { similarity function (eq. 1) }
$$

3: Select $m=k$ where $R_{i k}$ is minimum

4: Created and store $P_{i(n+1)}$ and $S_{i(n+1)}$

$$
P_{i(n+1)}=P_{i m}(X, Y), S_{(n+1)}=S_{i m}
$$

5: Build the contour $C T_{(n+1)}$ based on $P_{i(n+1)}$ array

6: Compute the centroid from of $C T_{(n+1)}$

7: if (slice set selected is empty) then end else goto 1 


\subsection{Manual Method}

The manual method uses gradient vector flow (GVF) snake [9, 10] for ICH segmentation, slice by slice. It is divided in two main tasks: first created the initial snake using a spline function (to obtain an approximation of ICH contour), and second the initial snake is deformed to produce the final segmentation.

The following algorithm described the method:

Algorithm 4. Manual Method

1: Select slice $I(n)$

2: Select Query Object

Extract the ICH contour (CTn). (Using spline)

3: GVF snake deformation $(C T n)$.

This method was designed (as control method) to produce perfect segmentation patterns which were used to validate semiautomatic methods.

\section{Results}

We have developed a set of three methods for object segmentation and tracking: 3D mathematical morphology based method (3DMM), similarity based method (SBM) and manual based method. These methods were applied successfully for segmentation and tracking of spontaneous ICH from CT head images. The algorithm prototypes were implemented in MATLAB. These prototypes were validated by medical researchers in a representative dataset of $36 \mathrm{CT}$ head images patient studies. Manual method was used as the control method to produce perfect (correct) segmentation patterns (see Fig.3). The performance of the semiautomatic methods was $83.3 \%, 30$ CT patient studies were segmented and tracked correctly. Both methods do not work correctly in $6 \mathrm{CT}$ patient studies (where the variation in the ICH shape among continuous slices was very high). Despite similar results were found concerning precision and robustness in $30 \mathrm{CT}$ patient studies; some differences were revealed in relation with user intervention, processing time and precision. The 3DMM has the

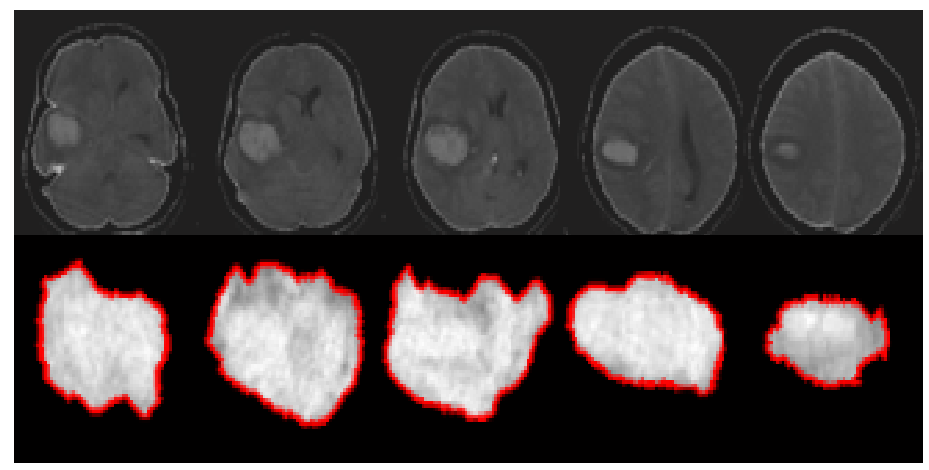

Fig. 3. Manual method results 


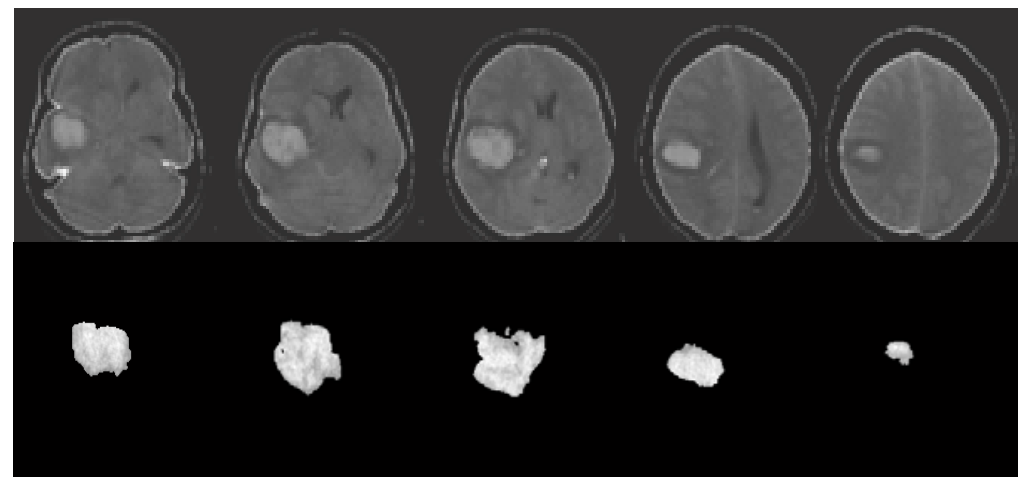

Fig. 4. Semiautomatic 3D mathematical morphology method results
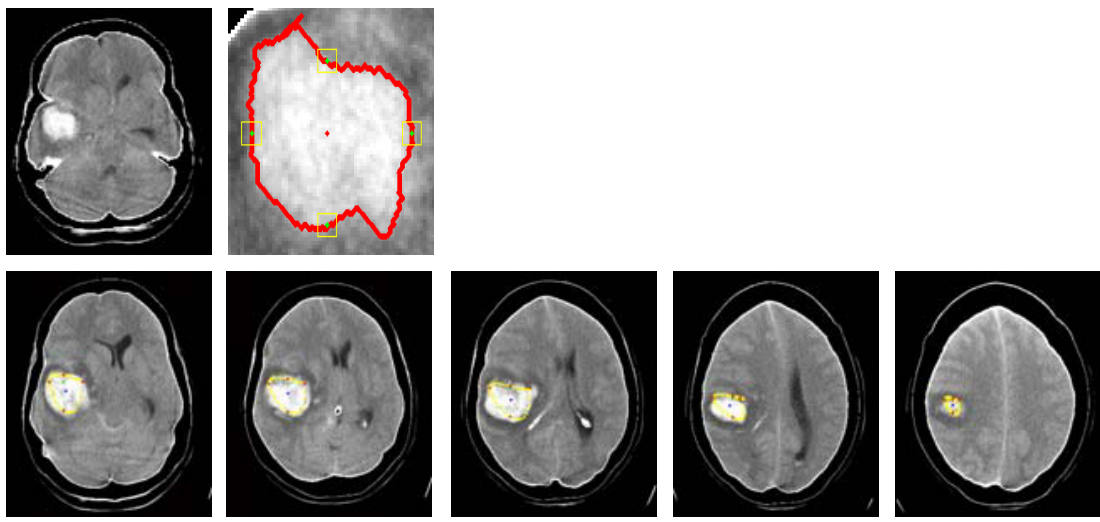

Fig. 5. Similarity method results. From top to bottom and left to right: First ICH slice, ICH query, ICH segmentation and tracking.

advantage to require the least user intervention and processing time, but it has the drawback to be the least precise (see Fig.4). SBM needed more user intervention and more processing time, but segmentation and tracking results achieved were more precise compared with 3DMM (see Fig.5).

\section{Conclusion}

In this paper we proposed a set of three methods for object segmentation and tracking. Two of these methods can be carryout in a semiautomatic way and one in a manual way (control method). These methods were applied successfully for segmentation and tracking of spontaneous ICH from CT head images. The efficiency of semiautomatic methods was confirmed on a representative dataset 36 patient studies, where these were capable to segment and track correctly the $\mathrm{ICH}$ in 30 patient studies $(83.3 \%)$. Compared with other approaches devoted to $\mathrm{ICH}$ segmentation [2, 3, 4, 11] our 
approach demonstrated similar or less computational complexity. The algorithms developed will be easily extended, with the corresponding adaptations, to solve others object recognition problems related or not with the medical image analysis area.

\section{Acknowledgments}

This work was carried out in the Center for Advanced Computer Sciences Technologies at Ciego de Avila University, Cuba. The authors thanks the Cuban Neurosciences Center for their financial support, as well as the Intensive Care Unit of Morón Hospital, Cuba for give us a representative set of patient images studies and their participation on the evaluation of the set of proposed methods performance.

\section{References}

1. Malkoff, A.E.-M.M.D.: Intracerebral Hemorrhage. The Internet Journal of Emergency and Intensive Care Medicine 5(1) (2001)

2. Cosic, D., Loncaric, S.: Computer System for Quantitative Analysis of ICH from CT head Images. In: 19th Annual International Conference of IEEE Engineering in Medicine and Biology Society, Chicago, USA (1997)

3. Loncaric, S., Dhawan, A.P., Cosic, D.: Quantitative intracerebral brain hemorrhage analysis. In: SPIE Medical Imaging, San Diego, USA (1999)

4. Majcenic, Z., Loncaric, S.: CT Image Labeling Using Simulated Annealing Algorithm. In: IX European Signal Processing Conference, Island of Rhodos, Greece (1998)

5. Liang, J., McInerney, T., Terzopoulos, D.: United Snakes. IEEE Trans. Medical Image Analysis 10, 215-233 (2006)

6. Falcão, A.X., Udupa, J.K., Miyazawa, F.K.: Ultra-fast user-steered segmentation paradigm: live-wire-on-the-fly. IEEE Trans. Medical Imaging 19(1), 55-62 (2000)

7. Llerena, Y.: Técnicas para el almacenamiento y recuperación de información gráfica en bases de datos de imágenes. In: CEIS, ISPJAE: Habana, Cuba (2003)

8. Fuertes, J.M.: Recuperación de Imágenes en bases de datos a partir del color y la forma, in E. T. S. de Ingeniería Informática. 1999, Universidad de Granada: Granada.

9. Xu, C., Prince, J.L.: Gradient Vector Flow. A New External Force for Snakes. In: IEEE Conf. on Comp. Vision Patt. Recog. Comp. Soc. Press, Los Alamitos (1997)

10. Xu, C., Prince, J.L.: Snake, shapes, and gradient vector flow. IEEE Trans. Image Processing, 359-369 (1998)

11. Majcenic, Z., Loncaric, S.: CT Image Labeling Using Simulated Annealing Algorithm. In: IX European Signal Processing Conference (1997) 